\title{
Éditorial
}

\section{Consommation d'alcool pendant la grossesse et trouble du spectre de l'alcoolisation fotale au Canada : qui, quoi, où?}

Jocelynn L. Cook, Ph. D. $(1,2,3)$

Diffuser cet article sur Twitter

\section{Comprendre le trouble du spectre de l'alcoolisation fotale}

La prévalence estimée du trouble du spectre de l'alcoolisation fœtale (TSAF) au Canada est supérieure à celles de l'autisme, de la paralysie cérébrale et de la trisomie 21 combinées. Le coût supplémentaire par cas de TSAF tout au long de la vie d'une personne est estimé à 1,1 million de dollars ${ }^{1}$.

Le TSAF est une incapacité permanente qui touche le cerveau et le corps des personnes exposées à l'alcool avant leur naissance. Souvent, ces personnes rencontrent des défis dans leur vie quotidienne et ont besoin d'un soutien sur le plan des habiletés motrices, de la santé physique, de l'apprentissage, de la mémoire, de la régulation des émotions et des habiletés socia$\operatorname{les}^{2,3}$. Selon des données récentes, le TSAF est associé à des maladies chroniques, à des troubles de santé mentale et à des expériences sociales négatives ${ }^{4,5}$. Lorsqu'elles ne reçoivent pas de diagnostic ni de soutien, les personnes atteintes de TSAF présentent des taux accrus de consommation problématique de substances, de problèmes de santé mentale, de démêlés avec la justice pénale, de prise en charge par les services de protection de l'enfance, de mauvais traitements psychologiques et physiques, de traumatismes et d'instabilité en matière de logement ${ }^{6}$.

Le TSAF découle de l'interaction entre plusieurs facteurs de risque et de vulnérabilité. Alors que toutes les populations qui consomment de l'alcool présentent un risque de TSAF, nous ne connaissons pas la fréquence de consommation d'alcool pendant la grossesse au Canada ni la prévalence du TSAF.

\section{Consommation d'alcool pendant la grossesse}

Le fait que l'alcool soit une substance tératogène neurocomportementale n'est ni controversé ni discutable. Pourtant, malgré des années de messages publics sur les méfaits de l'alcool pendant la grossesse et des millions de dollars consacrés aux campagnes de prévention, le nombre de femmes qui consomment de l'alcool pendant la grossesse n'a pas diminué ${ }^{\text {. }}$.

Que se passe-t-il exactement?

Nous savons que la vie des femmes qui consomment de l'alcool pendant leur grossesse est complexe. Nous savons que ces femmes consomment de l'alcool pour différentes raisons, que les déterminants sociaux de la santé jouent un rôle impor$\operatorname{tant}^{8}$ et que la stigmatisation a une incidence sur le signalement de la consommation d'alcool et l'accès aux interventions ${ }^{9}$. Par ailleurs, les femmes ne savent parfois pas qu'elles sont enceintes.

Il est à noter que, d'après la plus récente directive clinique sur le dépistage et les conseils en matière de consommation d'alcool pendant la grossesse, publiée en septembre 2020 par la Société des obstétriciens et gynécologues du Canada ${ }^{10}$, toutes les femmes enceintes devraient être interrogées sur leur consommation d'alcool. Si des habitudes de consommation sont établies, l'information doit être consignée dans le dossier médical du nourrisson après l'accouchement. Ces recommandations visent à ce que le dépistage et les conversations sur la consommation d'alcool soient intégrés dans les pratiques courantes et à ce que l'exposition soit notée dans les dossiers de la mère et de l'enfant. Les fournisseurs de soins de santé jouent un rôle essentiel dans la saisie de ces données et leur intégration dans les systèmes de données provinciaux et dans la normalisation des questions sur la consommation d'alcool et la création d'un environnement propice à des discussions fructueuses avec les patientes ou clientes.

\section{Surveillance}

Il existe peu de données de surveillance concernant l'alcool et la grossesse au Canada. Quelques rapports ont été publiés, et de nombreuses personnes ont travaillé d'arrache-pied en vue de recueillir des données courantes complètes sur l'alcool et la grossesse, au moyen d'approches rétrospectives et prospectives, au fil du temps et dans différentes administrations. Certaines enquêtes nationales ont fourni un aperçu de la consommation, dont l'Enquête canadienne sur l'expérience de la maternité (aujourd'hui abandonnée) et l'Enquête sur la santé dans les collectivités canadiennes (toujours active). Ces sources de données nous renseignent sur la quantité d'alcool que la personne déclare avoir consommée, mais nous ne disposons pas de données sur les raisons et les contextes de consommation, les difficultés ou obstacles qui freinent l'accès au soutien et aux services, les habitudes de consommation et le moment de la 
consommation pendant la grossesse. Les renseignements disponibles sont fondés sur des données autodéclarées dont les valeurs sont trop faibles, dans bien des cas, pour servir à l'établissement de conclusions générales.

Les bases de données et les registres provinciaux renferment parfois des informations sur la consommation d'alcool pendant la grossesse, mais ils dépendent des renseignements saisis dans la source de données (c.-à-d. les dossiers des patients). Bien que cette approche permette de recueillir l'essentiel des données sur la consommation d'alcool pendant la grossesse, des détails sont souvent omis et les jeux de données ont tendance à être incomplets. La consommation d'alcool est presque exclusivement autodéclarée et, si l'on considère les répercussions complexes de la stigmatisation et l'absence de dépistage systématique de la consommation d'alcool pendant la grossesse dans les formulaires prénataux, les données paraissent généralement peu fiables. On a tenté par le passé d'établir un dossier périnatal normalisé à l'échelle nationale, avec des données sur l'exposition prénatale, mais le projet semble au point mort.

De plus, le Canada ne dispose d'aucun ensemble d'indicateurs, et des données n’y sont pas recueillies de façon systématique et courante en ce qui a trait à la prévalence du TSAF. Encore une fois, le milieu de la recherche a fait un excellent travail en vue de recueillir des données sur la prévalence à partir de diverses études menées auprès de différentes populations au Canada. Nous avons des rapports provenant des communautés rurales ${ }^{11}$, des écoles urbaines ${ }^{12}$, des systèmes de protection de l'enfance ${ }^{13}$ et des systèmes de justice ${ }^{14}$. En 2019, l'Enquête canadienne sur la santé des enfants et des jeunes a tenté de recueillir des données sur le $\mathrm{TSAF}^{15}$. Ces études dépendent des résultats d'une évaluation diagnostique du TSAF, or l'accès des Canadiens aux services de diagnostic est nettement insuffisant pour répondre à la demande ${ }^{16}$. Il s'agit d'une lacune cruciale : si l'exposition prénatale à l'alcool n'est pas établie de façon précoce et saisie dans nos systèmes de données, nous perdons la possibilité d'entreprendre une surveillance précoce et d'offrir des conseils d'ordre préventif axés sur le soutien et les services. L'occasion de contribuer à l'atteinte du plein potentiel est ainsi manquée de façon tragique.
La saisie de données fiables dans les systèmes de données provinciaux sur l'exposition prénatale ou sur les habitudes de consommation et les quantités d'alcool consommées contribuerait grandement à l'étude de la prévalence, des tendances démographiques et régionales ainsi que des troubles concomitants. Nous pourrions alors repérer les périodes pendant lesquelles la consommation d'alcool et les troubles concomitants semblent en évolution - la pandémie de COVID-19 durant la dernière année en est un parfait exemple - afin de prévoir les adaptations à apporter aux programmes de prévention et de soutien. Ce type de données nous aiderait également à mieux comprendre l'efficacité des mesures de prévention et de réduction des méfaits en temps réel, ou du moins beaucoup plus rapidement que les années actuellement nécessaires pour produire des rapports sur les enquêtes nationales.

\section{Base de données nationale sur le TSAF}

La base de données nationale sur le TSAF est un autre projet national mis en œuvre pour recueillir des renseignements détaillés sur le TSAF, les facteurs de risque et l'évolution des patients. De 2010 à 2020, l'Agence de la santé publique du Canada a appuyé l'élaboration et la mise en œuvre de la base de données nationale sur le TSAF. NeuroDevNet et Kids Brain Health y ont contribué de 2013 à 2019. Le réseau CanFASD est maintenant le seul bailleur de fonds. La base de données vise à recueillir des renseignements sur le TSAF et sa prévalence dans toutes les cliniques de diagnostic du Canada.

À l'heure actuelle, nous disposons de plus de 3500 dossiers. Ceux-ci nous renseignent sur le contexte dans lequel les individus ont été exposés à l'alcool avant leur naissance, sur leur santé et sur leurs comportements. Ils nous permettent aussi de cerner les défis à surmonter afin que nous envisagions autrement la prévention du TSAF et le soutien aux femmes qui consomment de l'alcool pendant leur grossesse.

Malgré la capacité diagnostique limitée du Canada, nous pouvons déterminer, avec la pleine participation des cliniques à l'échelle du pays, la prévalence du TSAF au moyen d'un système comme celui de cette base de données nationale.

\section{Résumé et recommandation concernant le financement et le soutien}

La consommation d'alcool pendant la grossesse et le TSAF sont complexes, et nous devons comprendre l'ampleur du problème, de même que les situations connexes. Les systèmes de données provinciaux sont prêts à recueillir des données sur la consommation d'alcool, en particulier pendant la grossesse, ainsi que sur les habitudes de consommation et les quantités consommées. Il est essentiel que des questions sur ce sujet soient intégrées à la pratique courante des fournisseurs de soins de santé. La normalisation des formulaires périnataux serait également utile.

Le Canada a fait beaucoup de chemin et, à bien des égards, nous faisons partie des chefs de file dans le domaine de la recherche sur le TSAF. Cependant, en raison de notre système de santé fragmenté, les données recueillies présentent des disparités entre administrations et des obstacles freinent l'échange des données, ce qui rend difficile la production de rapports nationaux sur la consommation d'alcool pendant la grossesse et sur la prévalence du TSAF. Le perfectionnement de la base de données nationale sur le TSAF dans le cadre de l'infrastructure pancanadienne de surveillance, avec l'adhésion et la participation de toutes les administrations, rendrait possible une saisie efficace des données nationales et permettrait d'en rendre compte avec exactitude.

\section{Références}

1. Thanh NX, Jonsson E. Costs of fetal alcohol spectrum disorder in the Canadian criminal justice system. J Popul Ther Clin Pharmacol. 2015; 22(1):e125-131.

2. McLachlan K, Flannigan K, Temple V, Unsworth K, Cook JL. Difficulties in daily living experienced by adolescents, transition-aged youth, and adults with fetal alcohol spectrum disorder. Alcohol Clin Exp Res. 2020;44(8): 1609-1624. https://doi.org/10.1111 /acer. 14385

3. Skorka K, McBryde C, Copley J, Meredith PJ, Reid N. Experiences of children with fetal alcohol spectrum disorder and their families: a critical review. Alcohol Clin Exp Res. 2020; 44(6):1175-1188. https://doi.org/10 $.1111 /$ acer. 14335 
4. Lebel CA, Gibbard WB, Tortorelli C, et al. Prenatal Exposure And Child brain and mental Health (PEACH) study: protocol for a cohort study of children and youth with prenatal alcohol exposure. BMJ Open. 2021; 11(5):e051660. https://doi.org/10.1136 /bmjopen-2021-051660

5. Flannigan K, Kapasi A, Pei J, Murdoch I, Andrew G, Rasmussen C. Characterizing adverse childhood experiences among children and adolescents with prenatal alcohol exposure and fetal alcohol spectrum disorder. Child Abuse Negl. 2021;112:104888. https:// doi.org/10.1016/j.chiabu.2020.104888

6. Popova S, Temple V, Dozet D, O’Hanlon G, Toews C, Rehm J. Health, social and legal outcomes of individuals with diagnosed or at risk for fetal alcohol spectrum disorder: Canadian example. Drug Alcohol Depend. 2021; 219:108487. https://doi.org/10.1016/j .drugalcdep.2020.108487

7. Burd L. Drinking at the end of pregnancy: why don't we see it? Pediatr Res. 2020;88(2):142. https://doi.org /10.1038/s41390-020-0846-1

8. Terplan M. Alcohol-exposed pregnancy outcomes explained by social determinants of health. BJOG. 2015;122(13): 1739. https://doi.org/10.1111/1471 $-0528.13216$

9. Escañuela Sánchez T, Matvienko-Sikar K, Linehan L, O’Donoghue K, Byrne M, Meaney S. Facilitators and barriers to substance-free pregnancies in highincome countries: a meta-synthesis of qualitative research. Women Birth. 2021:S1871-5192(21)00076-7. https:// doi.org/10.1016/j.wombi.2021.04.010

10. Graves L, Carson G, Poole N, et al. Directive clinique no 405 : Dépistage et conseils en matière de consommation d'alcool pendant la grossesse. Journal d'obstétrique et gynécologie du Canada. 2020;42(9):1158-1173.e1. https://doi.org/10.1016/j.jogc.2020.07 .005

11. Robinson GC, Conry JL, Conry RF. Clinical profile and prevalence of fetal alcohol syndrome in an isolated community in British Columbia. CMAJ. 1987;137(3):203-207.
12. Popova S, Lange S, Poznyak V, et al. Population-based prevalence of fetal alcohol spectrum disorder in Canada. BMC Public Health. 2019;19(1):845. https://doi.org/10.1186/s12889-019 -7213-3

13. Popova S, Lange S, Burd L, Rehm J. Canadian children and youth in care: the cost of fetal alcohol spectrum disorder. Child Youth Care Forum. 2014;43(1):83-96. https://doi.org/10 .1007/s10566-013-9226-x

14. McLachlan K, McNeil A, Pei J, Brain U, Andrew G, Oberlander TF. Prevalence and characteristics of adults with fetal alcohol spectrum disorder in corrections: a Canadian case ascertainment study. BMC Public Health. 2019;19(1):43. https://doi.org/10.1186 /s12889-018-6292-X

15. Statistique Canada. Enquête canadienne sur la santé des enfants et des jeunes (ECSEJ) [Internet]. Ottawa (Ont.) : Statistique Canada [modification le 11 février 2019; consultation le 17 mai 2021]. En ligne à : https:// www23.statcan.gc.ca/imdb/p2SV_f .pl?Function $=$ getSurvey $\&$ SDDS $=5233$

16. Clarren SK, Lutke J, Sherbuck M. The Canadian guidelines and the interdisciplinary clinical capacity of Canada to diagnose fetal alcohol spectrum disorder. J Popul Ther Clin Pharmacol. 2011;18(3):e494-499. 